\title{
HYDRODYNAMIC MODELLING OF CIRCULATING FLUIDISED BEDS
}

\author{
J. J. NIEUWLAND, P. HUIZENGA, J. A. M. KUIPERS and W. P. M. van SWAAIJ \\ Twente University of Technology, Department of Chemical Engineering, PO Box 217, 7500 AE Enschede, \\ The Netherlands
}

(Received 12 May 1994; accepted for publication 4 October 1994)

\begin{abstract}
A one-dimensional model for the riser section of a circulating fluidised bed has been developed which describes the steady-state hydrodynamic key variables in the radial direction for fully developed axisymmetric flow. Both the gas and the solid phase are considered as two continuous media, fully penetrating each other. As a first approximation gas phase turbulence has been incorporated in our hydrodynamic model by applying a slightly modified version of the well-known Prandtl mixing length model. To solve the resulting set of transport equations, the solids distribution along the tube radius is required. Several strategies are given to obtain this information. In addition the effect of clusters on the momentum transfer between both phases has been modelled using an empirical correlation. Theoretically calculated results agree well with reported experimental data of different authors.
\end{abstract}

\section{INTRODUCTION}

Despite the fact that circulating fluidised beds (CFBs) find widespread application in the chemical and process industries for several decades (Yerushalmi and Avidan, 1985), their design and scale up is still very difficult which is mainly due to the complex hydrodynamic behaviour. Experimental investigations clearly demonstrated an inhomogeneous solids distribution in both the axial and radial directions (Kwauk et al., 1986; Bader et al., 1988; Miller and Gidaspow, 1992). The inhomogeneous solids distribution in the axial direction can be attributed to the acceleration of particles which enter the column at the bottom of the bed with low velocity. The inhomogeneous solids distribution in the radial direction, which is unfortunately less well understood, may cause significant downflow of particles near the tube wall. The nonuniform solids distribution and solids flow influences the particle residence time distribution, and thereby the reactor performance, to a large extent. Understanding and (a priori) prediction of the complex hydrodynamic behaviour is of crucial importance for developing processes involving CFBs.

This work is concerned with the development of a one-dimensional model which describes the steadystate hydrodynamics in radial direction for fully developed axi-symmetric flow.

\section{THEORETICAL MODEL}

\subsection{Governing equations}

In the present model the gas and solid phases are considered as two continuous media, fully penetrating each other. This assumption can also be justified for

\footnotetext{
To whom correspondence should be addressed.
}

the particulate phase due to the very high particle concentration in the systems of interest (Anderson and Jackson, 1967). For steady state, fully developed axi-symmetrical flow the velocities of both phases and the solids concentration do not change in the axial direction and in addition the radial velocities are equal to zero. With these assumptions the mass balances are satisfied automatically while the momentum balances for the gas and solid phase reduce respectively to:

$$
\begin{aligned}
& \frac{1}{r} \frac{\mathrm{d}}{\mathrm{d} r}\left(\epsilon_{\mathrm{f}} r \mu_{\mathrm{f}} \frac{\mathrm{d} u}{\mathrm{~d} r}\right)-\beta(u-v)-\epsilon_{\mathrm{f}}\left(\frac{\mathrm{d} P}{\mathrm{~d} z}-\rho_{\mathrm{f}} g\right)=0 \\
& \frac{1}{r} \frac{\mathrm{d}}{\mathrm{d} r}\left(\epsilon_{\mathrm{s}} r \mu_{\mathrm{s}} \frac{\mathrm{d} v}{\mathrm{~d} r}\right)+\beta(u-v)-\epsilon_{\mathrm{s}}\left(\frac{\mathrm{d} P}{\mathrm{~d} z}-\rho_{\mathrm{s}} g\right)=0
\end{aligned}
$$

To obtain the axial velocity profiles from eqs (1) and (2) constitutive relations are required which define the remaining variables in terms of the gas phase velocity $u$ and the solid phase velocity $v$. For fully developed flow the axial pressure gradient has a constant value and determines the superficial gas velocity.

\subsection{Constitutive relations}

- Solids distribution $\epsilon_{\mathrm{s}}(r)$. To solve eqs (1) and (2) the solid phase concentration $\epsilon_{\mathrm{s}}$ is required as a function of the radius. According to Matsen (1982) the solids distribution can be considered homogeneous for very dilute flows $(<0.5 \%)$, where its value can be related to the overall mass flux. For more dense systems empirical correlations, reported in the literature (Zhang et al., 1991; Rhodes et al., 1992), can be used to describe the inhomogeneous solids distribution. One of these empirical correlations has been developed by 
7hang et al. (1991). In their study the particle properties, equipment geometries and operating conditions were varied systematically over a wide range of interest. Zhang et al. concluded that in all cases the radial solids distribution only depended on the cross-sectional averaged solids concentration $\overline{c_{s}}$ and the dimensionless radial coordinate $\theta$ :

$$
\epsilon_{\mathrm{s}}(\theta)=1-\left(1-\overline{\epsilon_{\mathrm{s}}}\right)^{0.191+\theta^{2.5}+3 \theta^{11}}
$$

Unfortunately, these authors calculated the average solids concentration $\overline{\epsilon_{\mathrm{s}}}$ from the experimentally determined axial pressure gradient using the well-known manometer formulae. Due to the neglect of wall friction the results of Zhang et al., as given by eq. (3), are probably not valid for very dilute systems with high velocities. Furthermore, the average solids concentration $\overline{\epsilon_{\mathrm{s}}} \mathrm{c}$ calculated from eq. (3) and its definition equation:

$$
\overline{\epsilon_{\mathrm{s}}} \mathbf{c}=\int_{0}^{1} 2 \theta \epsilon_{\mathrm{s}}(\theta) \mathrm{d} \theta
$$

differ slightly from the cross-sectional averaged solids concentration $\overline{\epsilon_{\mathrm{s}}}$ appearing in eq. (3) (see Table 1).

Rhodes et al. (1992) also reported an inhomogeneous solids distribution which could be described in terms of the dimensionless radial position $\theta$ and the cross-sectional averaged solid phase concentration as follows:

$$
\epsilon_{\mathrm{s}}(\theta)=2 \overline{\epsilon_{\mathrm{s}}} \theta^{2}
$$

As evident from inspection of eq. (5), a zero solids concentration in the tube centre is predicted which is contradictory to experimental observations (Dry, 1986; Miller and Gidaspow, 1992). In this work, therefore, eq. (3) is used in which the value of $\overline{\epsilon_{s}}$ is (iteratively) determined from the specified solids mass flux:

$$
G_{\mathrm{s}}=\int_{0}^{1} 2 \theta \epsilon_{\mathrm{s}}(\theta) \rho_{\mathrm{s}} v(\theta) \mathrm{d} \theta
$$

Another possibility of obtaining the radial solids distribution is the use of experimental data.

- Interphase momentum transfer coefficient $\beta$. In systems of interest, i.e. in which $\epsilon_{\mathrm{s}}<0.2$, the interphase momentum transfer coeflicient $\beta$ can be derived from the correlation obtained by Wen and Yu (1966):

Table 1 . Comparison of calculated average solids concentration $\bar{\epsilon}_{\mathrm{s}}^{c}$ [eq. (4)] and cross-sectional averaged solids concentration $\overline{\epsilon_{s}}$ appearing in eq. (3)

\begin{tabular}{lll}
\hline$\overline{\epsilon_{\mathrm{s}}}$ & $\overline{\epsilon_{\mathrm{q}}}$ & $\overline{\epsilon_{\mathrm{c}}^{\mathrm{c}}} / \overline{\epsilon_{\mathrm{s}}}$ \\
\hline 0.0100 & 0.0109 & 1.0915 \\
0.0500 & 0.0535 & 1.0702 \\
0.1000 & 0.1045 & 1.0448 \\
0.2000 & 0.1995 & 0.9977 \\
\hline
\end{tabular}

$$
\beta=\frac{3}{4} C_{d_{s} s} \frac{\epsilon_{\mathrm{f}}\left(1-\epsilon_{\mathrm{f}}\right)}{d_{p}} \rho_{\mathrm{f}}|\bar{u}-\bar{v}| \epsilon_{\mathrm{f}}^{-2.65}
$$

where the drag coefficient for an isolated particle $C_{d, s}$ depends on the Reynolds number as follows (Schiller and Naumann, 1935):

$$
C_{d, s}= \begin{cases}\frac{24}{R e_{\mathrm{p}}}+\frac{3.6}{R e_{\mathrm{p}}^{0.313}} & R e_{\mathrm{p}}<1000 \\ 0.44 & R e_{\mathrm{p}} \geq 1000\end{cases}
$$

- Gas phase viscosity $\mu_{\mathrm{f}}$. Because the Reynolds number based on the tube diameter in the fast fluidisation regime equals typically $10,000-100,000$, the gas flow will be turbulent. The particles which are present influence the turbulence behaviour of the gas phase. Un the basis of experimental observations, Tsuji et al. (1984) reported that large, heavy particles tend to increase the turbulence intensity, while small particles decrease the turbulence intensity. The particles used in this work can be classified as small (average particle diameter $\left.d_{p}=50 \mu \mathrm{m}\right)$, and on the basis of the results obtained by Tsuji et al, it is expected that the turbulence intensity will be moderated due to the presence of particles.

The gas phase viscosity $\mu_{\mathrm{f}}$ is taken as the sum of the (constant) gas phase shear viscosity and an eddy viscosity to account for the turbulent momentum transfer. The eddy viscosity is given by a modified Prandtl mixing length model:

$$
\mu_{\mathrm{t}}=\rho_{\mathrm{f}} K^{2}(R-r)^{2}\left|\frac{\mathrm{d} u_{\mathrm{z}}}{\mathrm{d} r}\right| f\left(\epsilon_{\mathrm{f}}\right)
$$

where the function $f\left(\epsilon_{\mathrm{f}}\right)$ corrects for the presence of particles. For the function $f\left(\epsilon_{\Gamma}\right)$, as a first approximation, a simple linear form has been chosen which equals 1 for single phase flow $\left(\epsilon_{\mathrm{f}}=1\right)$ and 0 for flow through a bed at incipient fluidisation $\left(\epsilon_{\mathrm{f}}=\epsilon_{\mathrm{f}, \mathrm{mf}}\right)$ :

$$
f\left(\epsilon_{\mathrm{f}}\right)=\frac{\epsilon_{\mathrm{F}}-\epsilon_{\mathrm{f}, \mathrm{mf}}}{1-\epsilon_{\mathrm{f}, \mathrm{mf}}}
$$

For single phase turbulent flow the empirical parameter $K$ has the value 0.36 (Deissler, 1955).

- Solid phase shear viscosity $\mu_{\mathrm{s}}$. For the solid phase shear viscosity, as a first approximation, a constant value of 0.724 Pas has been taken, although reality is much more complex (Siemes and Hellmer, 1962; Grace, 1970). The value of $0.724 \mathrm{~Pa}$ s has been reported by Gidaspow et al. (1989) for spent catalyst $\left(d_{p}=76 \mu \mathrm{m}\right.$, $\rho_{\mathrm{s}}=1714 \mathrm{~kg} \mathrm{~m}^{-3}$ ) in the fast fluidisation regime, i.e. the system of interest. Gidaspow et al. calculated this viscosity from the published experimental data of Bader et al. (1988), using the following integral momentum balance:

$\epsilon_{\mathrm{s}, w} \mu_{\mathrm{s}}\left(\frac{\mathrm{d} v}{\mathrm{~d} r}\right)_{\mathrm{w}}=\frac{1}{4} D\left[\left(\frac{\Delta P}{L}\right)-\left(\overline{\epsilon_{\mathrm{f}}} \rho_{\mathrm{f}}+\overline{\epsilon_{\mathrm{s}}} \rho_{\mathrm{s}}\right) g+f_{\mathrm{f}, \mathrm{w}}\right]$ 
In this equation $f_{\mathrm{f}}$ w denotes the wall friction of the fluidum phase for which Gidaspow et al. used a relation given by Arastoopour and Gidaspow (1979).

Computational experience has shown that the exact value of the solid phase shear viscosity is not critical, as long as the correct order of magnitude is used.

\subsection{Boundary conditions}

In the tube centre the viscous momentum flows should vanish for both phases, resulting in the following Neumann type boundary conditions:

$$
\begin{aligned}
& \lim _{r \rightarrow 0} \epsilon_{\mathrm{f}} \mu_{\mathrm{f}} r \frac{\mathrm{d} u}{\mathrm{~d} r}=0 \\
& \lim _{r \rightarrow 0} \epsilon_{\mathrm{s}} \mu_{\mathrm{s}} r \frac{\mathrm{d} v}{\mathrm{~d} r}=0
\end{aligned}
$$

With respect to the boundary conditions imposed at the tube wall the situation is more complex and will be discussed subsequently in more detail. Imposing the traditional no-slip condition for both phases resulted in a discontinuity in the radial profile of the slip velocity in the neighbourhood of the tube wall. The occurrence of this physically unrealistic phenomenon can be understood by considering the mechanism of radial momentum transport in the gas phase in more detail. Due to the faet that the eddy viscosity vanishes at the tube wall, momentum transport in radial direction will occur through the remaining molecular mechanism in the vicinity of the wall. However, the molecular shear viscosity is very small in comparison with the eddy viscosity, which would cause a very large velocity gradient in the radial direction due to the requirement of the continuity of the total (i.e. sum of laminar and turbulent contributions) momentum flux. The solid phase shear viscosity is four orders of magnitude larger than the molecular shear viscosity in the gas phase and therefore effective (i.e. without requiring large radial velocity gradients) momentum transport in the radial direction could be provided by the solid phase instead of the gas phase. This alternative mode of momentum transport to the tube wall is only possible if a sufficiently large velocity difference between both phases develops. However, at the tube wall the slip velocity is forced to zero due to the imposed no-slip boundary conditions for both phases. That is the reason for the observed discontinuity in the vicinity of the tube wall. An alternative boundary condition for the solid phase has been proposed by Ding and Gidaspow (1990). In their expression the axial solids velocity at the tube wall is taken as proportional to the axial solids velocity gradient at the tube wall. They postulated that the proportionality constant equals the mean distance between the particles. Applying their expression, the same discontinuity in the slip velocity near the tube wall has been observed due to an underestimate of the slip velocity. As reported by Ding and Gidaspow the boundary condition approaches the no-slip boundary condition for small particles.

The physically unrealistic behaviour can be suppressed by using an alternative boundary condition for the solid phase and has been obtained from the reduced gas phase momentum equation in which the net radial momentum transport has been neglected:

$$
\beta(u-v)=-\epsilon_{\mathrm{f}}\left(\frac{\mathrm{d} P}{\mathrm{~d} z}-\rho_{\mathrm{f}} g\right)
$$

It should be noted that this equation allows for the slip of solids along the tube wall. For the gas phase the traditional no-slip condition has been applied at the tube wall:

$$
u=0
$$

This combination of boundary conditions, imposed at the tube wall, eliminated the earlier observed discontinuity in the radial profile of slip velocity.

\section{RESULTS}

Figure 1 shows a comparison between the theoretically calculated and experimentally determined (van Breugel et al., 1969a) axial solid phase velocity profile for a superficial gas velocity of $6.3 \mathrm{~m} \mathrm{~s}^{-1}$ and a solids mass flux of $390 \mathrm{~kg} \mathrm{~m}^{-2} \mathrm{~s}^{-1}$. For the radial solids distribution use has been made of the experimental data reported by van Breugel et al. (see Fig. 2). To study the effect of incorporating turbulence two cases were considered: one with and one without modelling turbulence. It may be clear from Fig. 1 that modelling the turbulent momentum transport is necessary to forecast correct velocity profiles. The significance of this mechanism for radial momentum transport also follows from Fig. 3, showing a comparison with experimental data (Yang et al., 1992) for dilute gas-solids flow. In this case the radial solids distribution has been obtained from the empirical formula of Zhang et al. (eq. (3) in this paper). Figure 4 shows the calculated radial slip velocity profile (i.e. the difference between gas phase velocity and solid phase velocity). The observed maximum near the tube wall, which has also been observed experimentally by Yang et al., could only be reproduced in the case where the turbulent momentum transport in the gas phase was accounted for. This local maximum was also found for a case with homogeneous solids distribution and for a case in which the no-slip condition was imposed for both phases, which leads to the conclusion that the observed phenomenon is not a result of solids segregation in radial direction or from applying the new boundary condition at the tube wall. The existence of the maximum can be attributed to the strong decrease in the turbulent momentum transport in the radial direction in the vicinity of the tube wall due to the presence of the factor $(R-r)^{2}$ in eq. (9). When the solids distribution is uniform it is clear that in this region, with increasing radius, the total gas phase viscosity decreases sharply resulting in less effective momentum transport in the radial direction. 


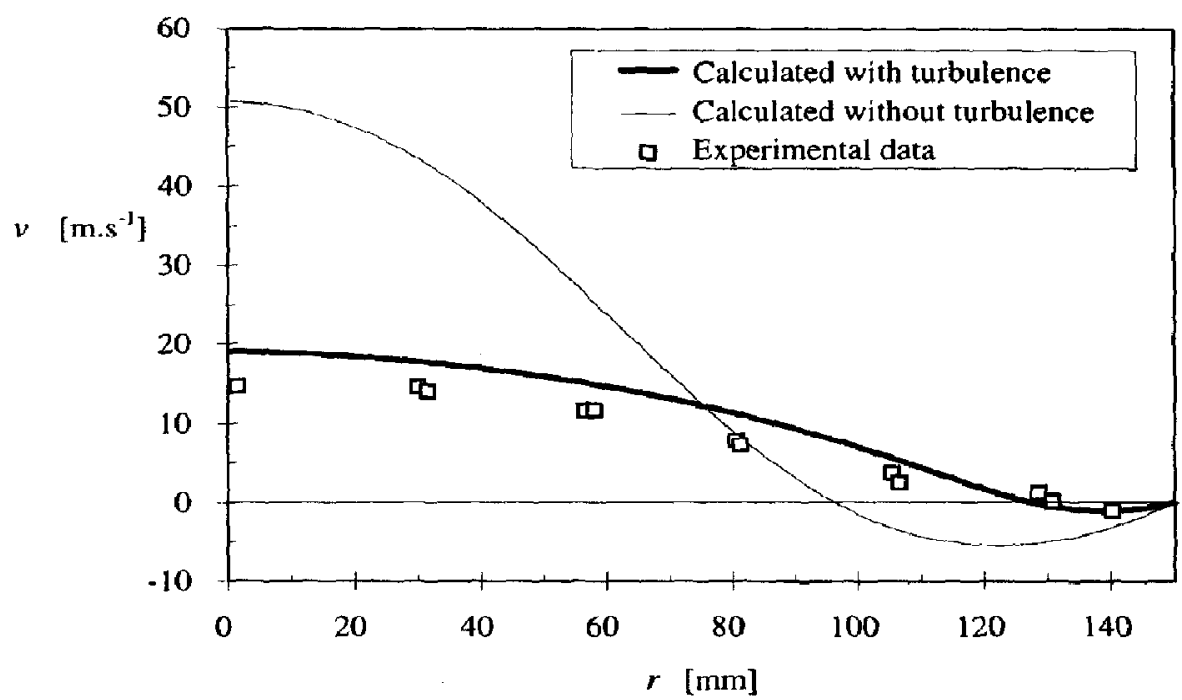

Fig. 1. Theoretically calculated and experimentally determined (van Breugel $e x$ al., 1969a) axial solids velucity profile; $D=0.30 \mathrm{~m}, U=6.3 \mathrm{~ms}^{-1}, G_{\mathrm{q}}=390 \mathrm{~kg} \mathrm{~m}^{-2} \mathrm{~s}^{-1}, d_{p}=40 \mu \mathrm{m}, \rho_{\mathrm{s}}=2300 \mathrm{~kg} \mathrm{~m}^{-3}$.

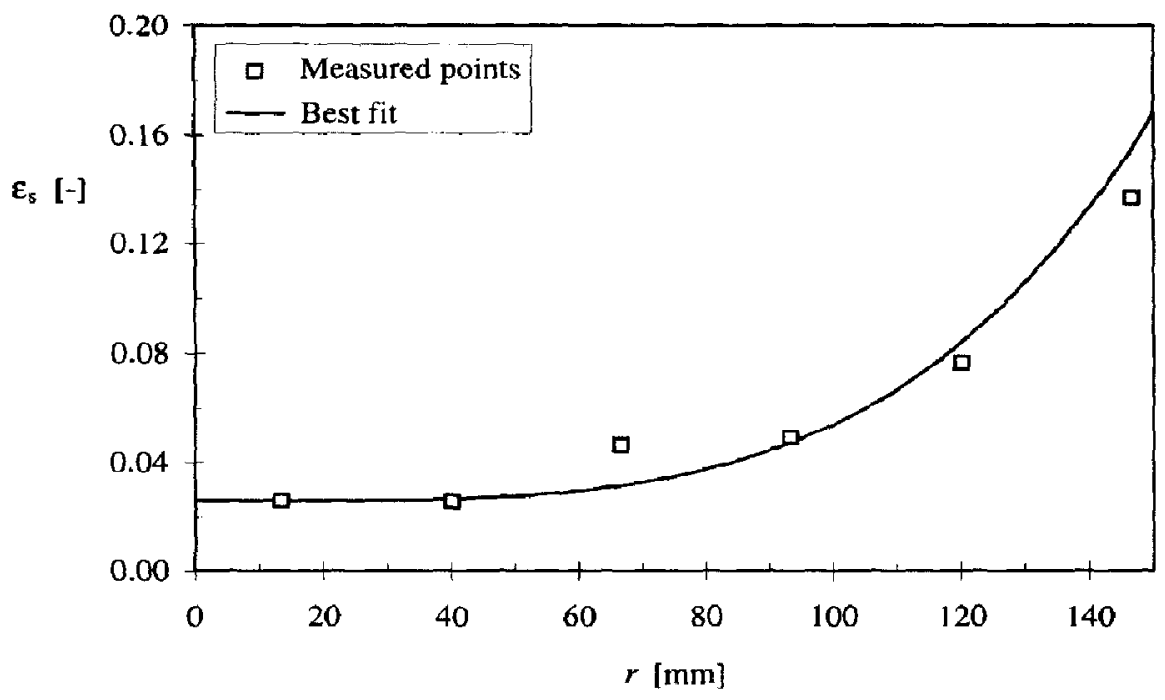

Fìg, 2. Experimentally determined (van Breugel et al, 1969a) solidity profile; $D=0.30 \mathrm{~m}, \quad U=6.3 \mathrm{~ms}$, $G_{\mathrm{s}}=390 \mathrm{~kg} \mathrm{~m}^{-2} \mathrm{~s}^{-1}, d_{p}=40 \mu \mathrm{m}, \rho_{\mathrm{s}}=2300 \mathrm{~kg} \mathrm{~m}^{-3}$.

However, due to its comparatively high value for the shear viscosity the solid phase is more effective with respect to this momentum transport and as a consequence a velocity difference develops to enable momentum transfer from the gas phase to the solids phase. Closer to the wall the turbulent part of the gas phase viscosity vanishes, and the constant laminar part remains. Due to the increasing radial gradient of the gas phase velocity close to the tube wall, the contribution of the solid phase is of less importance and consequently a smaller slip velocity results. Thus in this case the net force (i.e. pressure force minus gravity force) acting on a gas phase volume element can effectively be transmitted to the tube wall.

For an inhomogeneous solids distribution there exists a certain radial position where the pressure force equilibrates the gravity force acting on both phases. To the left of this position the pressure force exceeds the gravity force, resulting in an increasing momentum flow rate in the radial direction. At the right side of the aforementioned position of equilibrium the pressure force is insufficient to support both phases and the radial momentum transport has to provide additional momentum. These two effects 


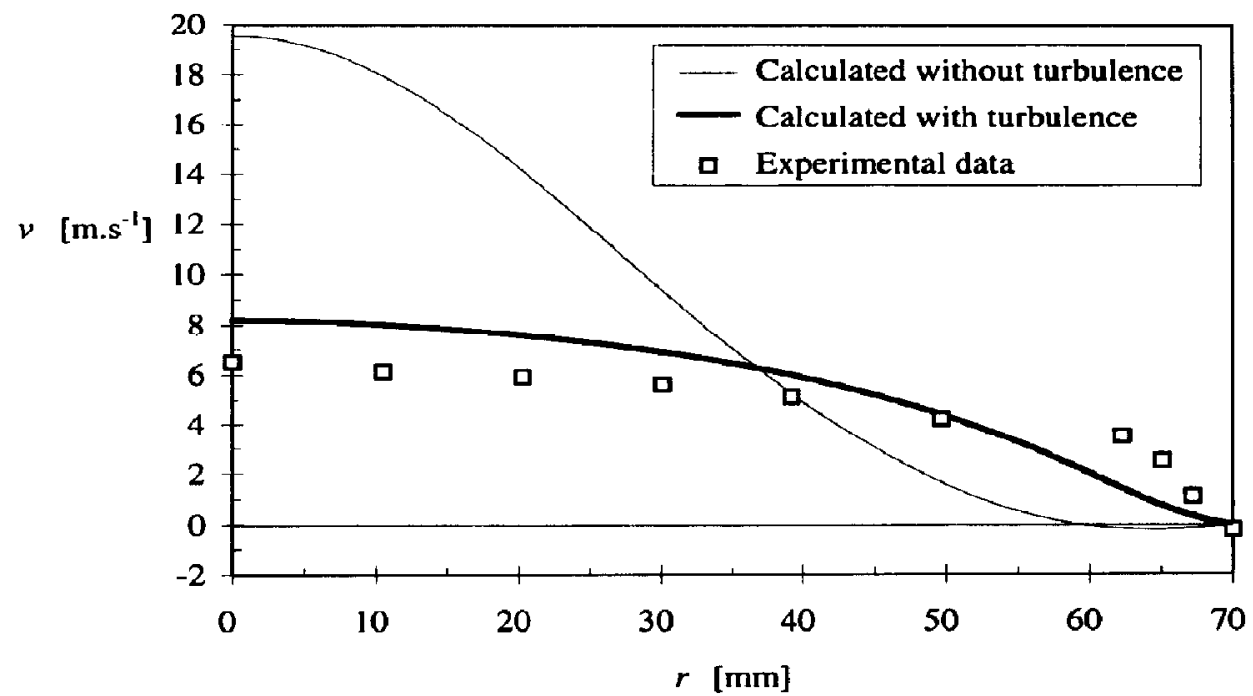

Fig. 3. Theoretically calculated and experimentally determined (Yang et al., 1992) axial solids velocity profile; $D=0.14 \mathrm{~m}$, $U=4.333 \mathrm{~m} \mathrm{~s}^{-1}, G_{\mathrm{s}}=22 \mathrm{~kg} \mathrm{~m}^{-2} \mathrm{~s}^{-1}, d_{p}=54 \mu \mathrm{m}, \rho_{\mathrm{s}}=1545 \mathrm{~kg} \mathrm{~m}^{-3}$.

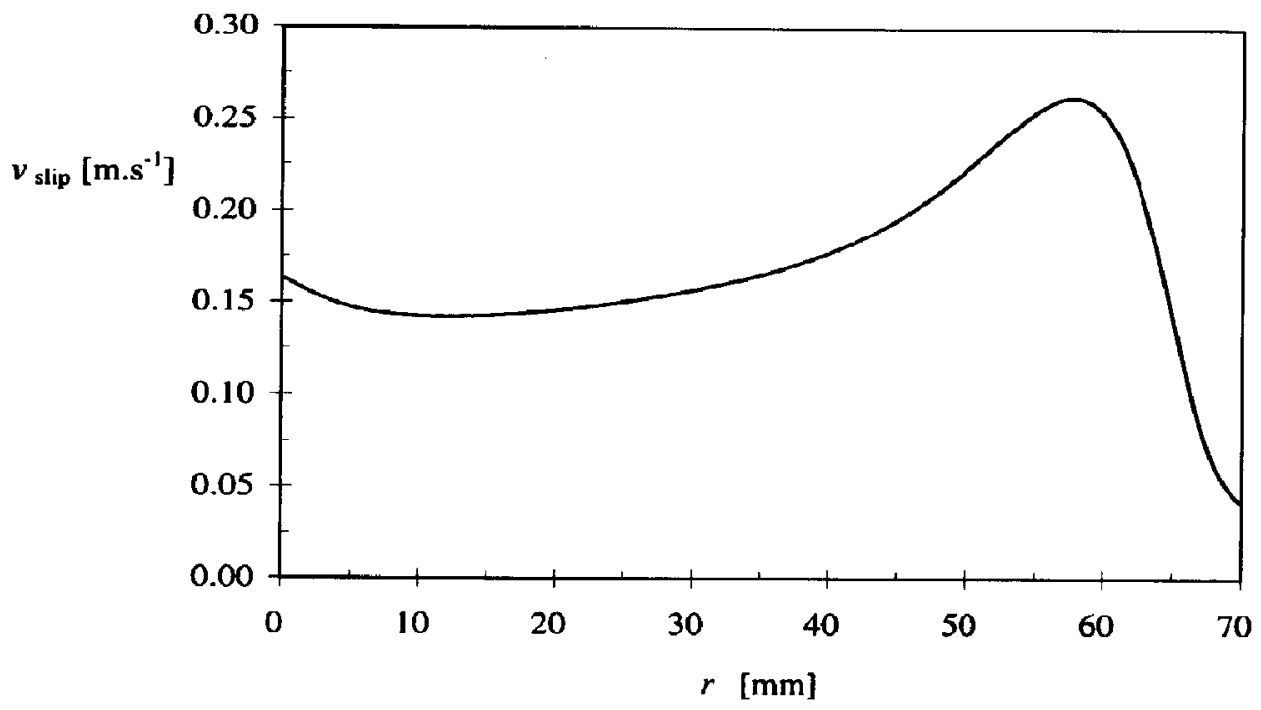

Fig. 4. Theoretically calculated slip velocity profile; $D=0.14 \mathrm{~m}, U=4.33 \mathrm{~m} \mathrm{~s}^{-1}, G_{\mathrm{s}}=22 \mathrm{~kg} \mathrm{~m}^{-2} \mathrm{~s}^{-1}, d_{p}=54 \mu \mathrm{m}$, $\rho_{\mathrm{s}}=1545 \mathrm{~kg} \mathrm{~m}^{-3}$.

cause an inflection point in the velocity profiles of both phases (Fig. 1) which does not exactly coincide with the aforementioned equilibrium position due to the increasing area with increasing distance from the centre on one hand and an increase in the suspension viscosity caused by the higher solids concentration on the other hand. At the right side of this inflection point the turbulent part of the gas phase viscosity decreases due to both the factor $(R-r)^{2}$ and the decreasing velocity gradient. Because the gas phase contribution to the radial momentum transport decreases, the solid phase contribution increases, resulting in a higher slip velocity. Closer to the tube wall the solids concentration increases and consequently an augmented part of the pressure force acts on the solid phase. This effect causes a decreased momentum transfer rate from the gas phase to the solids phase, resulting in a maximum in the slip velocity profile. The slip velocity profile exhibits an increase in the centre of the tube, less well pronounced however, due to the vanishing turbulent part of the gas phase viscosity and the flattening of the velocity profile.

Experimental observations (Monceaux et al., 1986; Miller and Gidaspow, 1992; Yang et al., 1992) have shown that velocity profiles have a more parabolic 


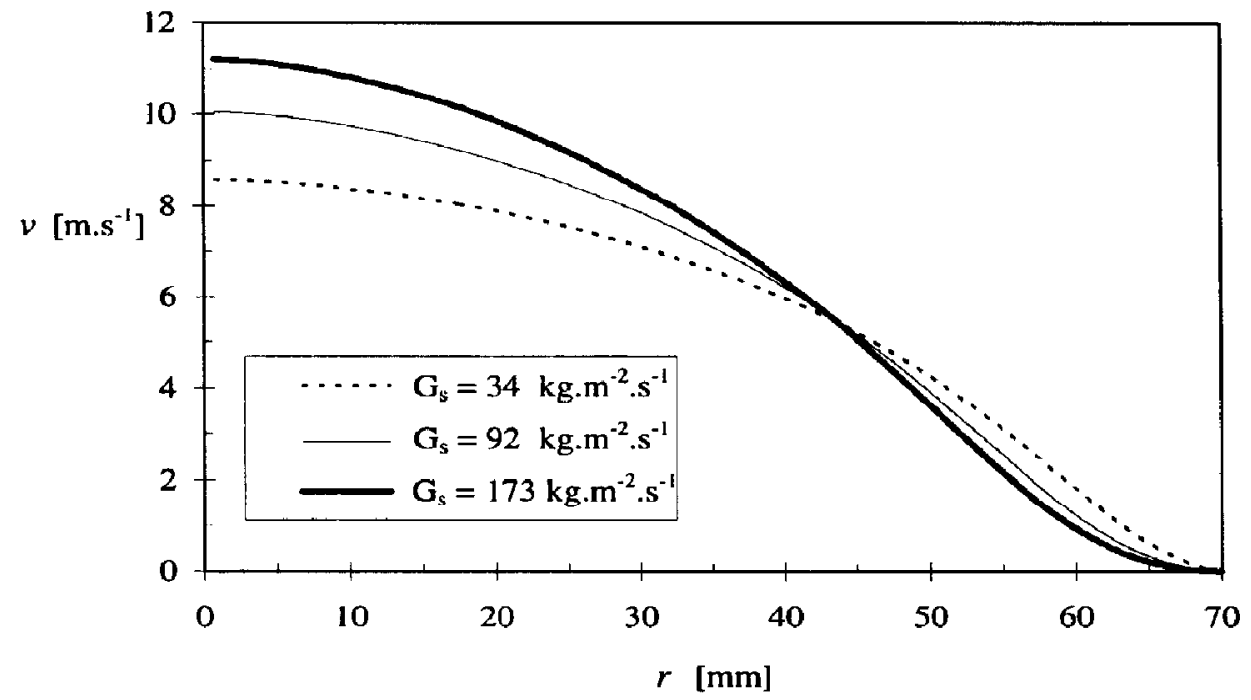

Fig. 5. Theoretically calculated axial solid phase velocity profiles for different solid mass fluxes; $D=0.14 \mathrm{~m}, U=4.33 \mathrm{~m} \mathrm{~s}{ }^{-1}$, $d_{p}=54 \mu \mathrm{m}, \rho_{\mathrm{s}}=1545 \mathrm{~kg} \mathrm{~m}^{-3}$.

shape at higher solid fluxes. Figure 5, depicting the calculated axial solid velocity profiles as a function of radius for different solid fluxes, demonstrates the capability of the theoretical model to predict this experimentally observed phenomenon.

\section{DISCUSSION}

Figures 1 and 3 show good agreement between theoretically calculated and experimentally determined axial velocity profiles. Theoretically calculated slip velocities, which are of the order of the terminal velocity of a single particle, are considerably smaller than the experimentally determined slip velocities which can be as high as $\mathbf{3 0}$ times the terminal velocity (van Breugel et al., 1969a). These high slip velocities are attributed to the formation of clusters of particles, as experimentally observed by Yerushalmi et al. (1978) and made plausible by Grace and Tuot (1979) on the basis of a linear stability analysis of the hydrodynamic equations of change. Figure 6 shows experimentally determined local slip velocity profiles as a function of the radius, measured by Yang et al. (1992), for particles having a terminal velocity of $0.12 \mathrm{~m} \mathrm{~s}^{-1}$. From this figure it can be concluded that the slip velocity increases with increasing solid phase mass fluxes. An estimation of the mean cluster size $d_{\mathrm{cl}}$ can be made assuming the clusters to be small spheres with a cluster voidage $\epsilon_{\mathrm{cl}}$, which equals the incipient fluidisation porosity. The results of these calculations are shown in Table 2.

As a first approximation it is assumed that the ratio of the local slip velocity and the terminal velocity of a single particle only depends on the solids concentration, following the approach of Matsen (1982) for dilute systems $\left(\epsilon_{\mathrm{s}}<0.01\right)$. Figure 7 shows the reduced slip velocity, i.e. the local slip velocity divided by the terminal velocity of a single particle, as a function of the solids concentration according to the experimental data of van Breugel et al. (1969a). The data shown in Fig. 7 have been derived from their experiments at several gas velocities and solid phase mass fluxes $\left(d_{p}=40 \mu \mathrm{m}, \rho_{\mathrm{s}}=2,300 \mathrm{~kg} \mathrm{~m}^{-3}\right)$. On the basis of these data an empirical correlation has been developed which yields a reduced slip velocity of one for zero solids concentration:

Table 2. Calculated cluster parameters from experimentally determined slip velocities; $d_{p}=54 \mu \mathrm{m}, \rho_{\mathrm{s}}=1,545 \mathrm{~kg} \mathrm{~m}^{-3}$ and $\epsilon_{\mathrm{cl}}=0.402$

\begin{tabular}{lccc}
\hline Numbers of particles in cluster & $\begin{array}{l}d_{\mathrm{el}} \\
(\mu \mathrm{m})\end{array}$ & $\begin{array}{l}\mathrm{V}_{\text {term,cl }} \\
\left(\mathrm{m} \mathrm{s}^{-1}\right)\end{array}$ & $V_{\text {term,cl }} / \mathrm{V}_{\text {term, }}$ \\
\hline & 54 & 0.1195 & 1.0 \\
150 & 236 & 0.8081 & 6.8 \\
250 & 403 & 1.5173 & 12.7 \\
500 & 508 & 1.9297 & 16.1 \\
750 & 582 & 2.2066 & 18.5 \\
1,000 & 640 & 2.4206 & 20.3 \\
\hline
\end{tabular}




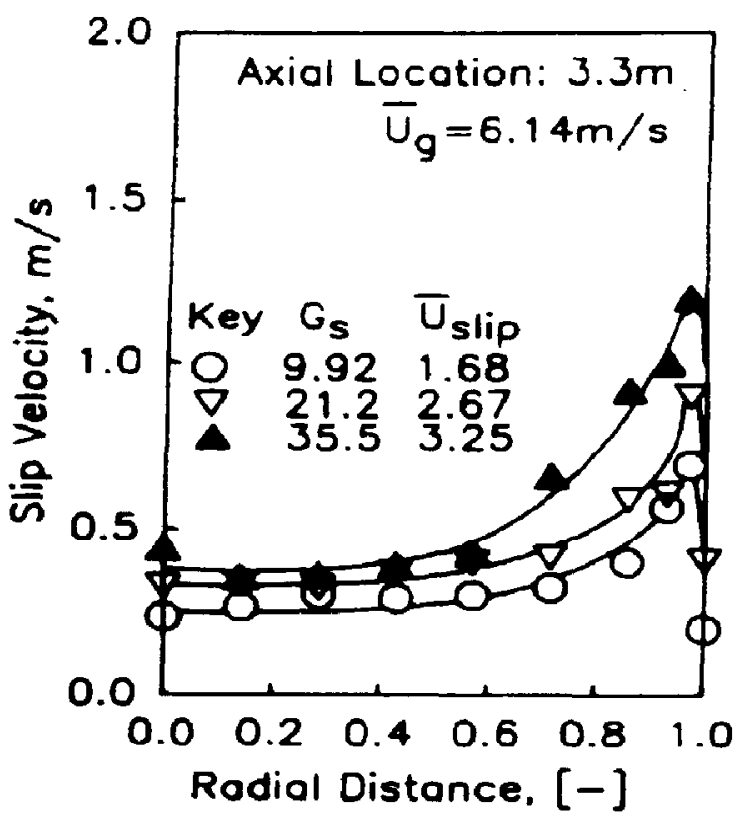

Fig. 6. Experimentally determined slip velocity profiles (Yang $e t$ al., 1992) for different solid phase mass fluxes; $D=0.14 \mathrm{~m}, U=6.14 \mathrm{~m} \mathrm{~s}^{-1}, d_{p}=54 \mu \mathrm{m}, \rho_{\mathrm{s}}=1545 \mathrm{~kg} \mathrm{~m}^{-3}$.

$\frac{v_{\text {slip }}}{v_{\text {term }}}= \begin{cases}0.997+442.35 \epsilon_{\mathrm{s}}-1733.42 \epsilon_{\mathrm{s}}^{2} & \epsilon_{\mathrm{s}}<0.1276 \\ 29.22 & \epsilon_{\mathrm{s}} \geq 0.1276 .\end{cases}$

Due to the scatter of the data at higher solids concentrations, a constant reduced slip velocity has been assumed for $\epsilon_{\mathrm{s}} \geq 0.1276$. Expression (16) can be used to quantify the less efficient momentum transfer due to the existence of clusters. According to Wen and $\mathrm{Yu}$ (1966) the ratio of the forces acting on a single isolated particle and a single particle surrounded by other particles equals the ratio of the terminal velocity and the slip velocity as long as the Reynolds number, based on the local superficial slip velocity and particle diameter, is smaller than 2 . Therefore, the term $\epsilon^{-2.65}$ in eq. (7), which is a correction function due to the presence of other particles, has been replaced by:

$$
g(\epsilon)=\frac{v_{\text {term }}}{v_{\text {slip }}}
$$

Although relation (16) has been derived for the particles used by van Breugel et al. $\left(d_{p}=40 \mu \mathrm{m}, \rho_{\mathrm{s}}=2300 \mathrm{~kg} \mathrm{~m}^{-3}\right)$ it follows from Figs 8 and 9 that this relation is, in addition, capable of describing the slip velocity in satisfactory agreement with the experimental data reported by Yang et al. (1992). In both cases the agreement between the calculated velocity profiles of the single phases and the experimental data was only slightly influenced by applying relation (16).

\section{CONCLUSION}

A one dimensional model has been developed which describes the steady state hydrodynamics of fully developed axi-symmetrical riser flow. In this model the turbulent momentum transport has been modelled using a slightly modified Prandtl mixing length model. The radial solids distribution has been obtained from experimental data and empirical correlations. Calculated axial velocity profiles appeared to be in good agreement with experimental data for both dilute and dense riser flow. An empirical correlation was developed and incorporated in the hydrodynamic model to correct the interphase momentum

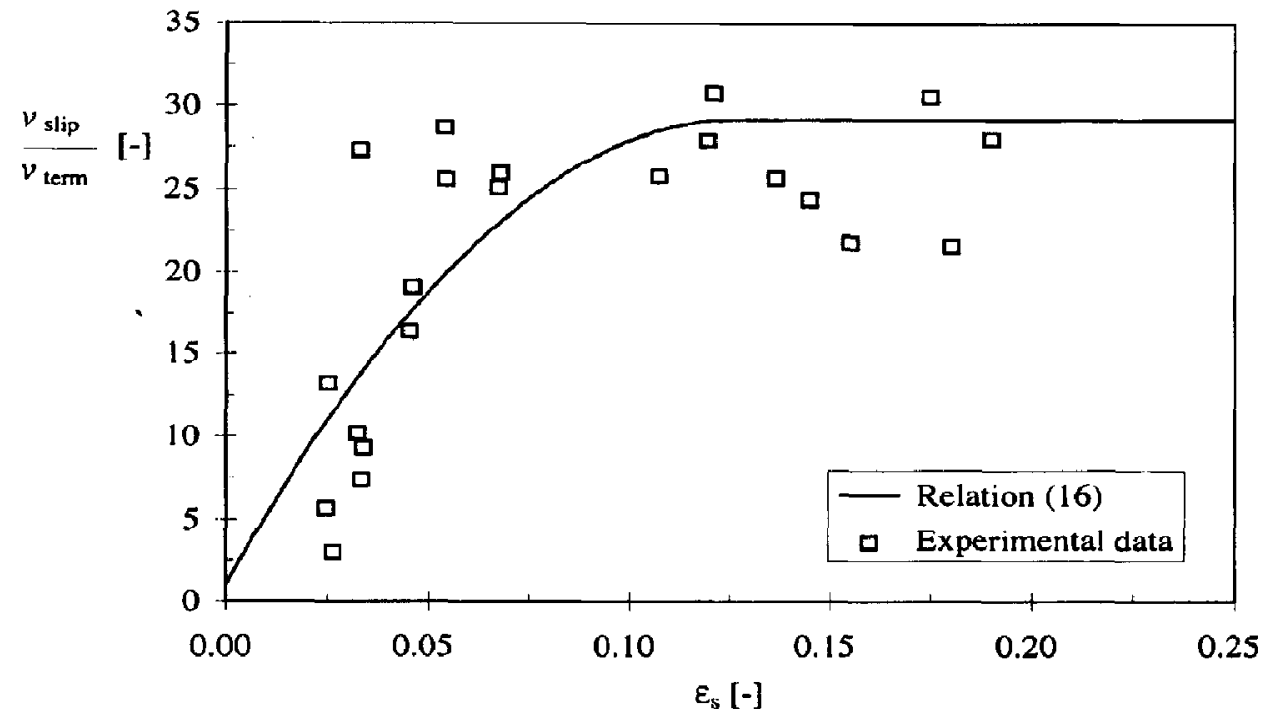

Fig. 7. Reduced slip velocity (slip velocity divided by terminal velocity) as a function of solids concentration $\epsilon_{\mathrm{s}}$ computed from data of van Breugel et al. (1969a); $D=0.30 \mathrm{~m}, d_{p}=40 \mu \mathrm{m}, \rho_{\mathrm{s}}=2300 \mathrm{~kg} \mathrm{~m}^{-3}$. 


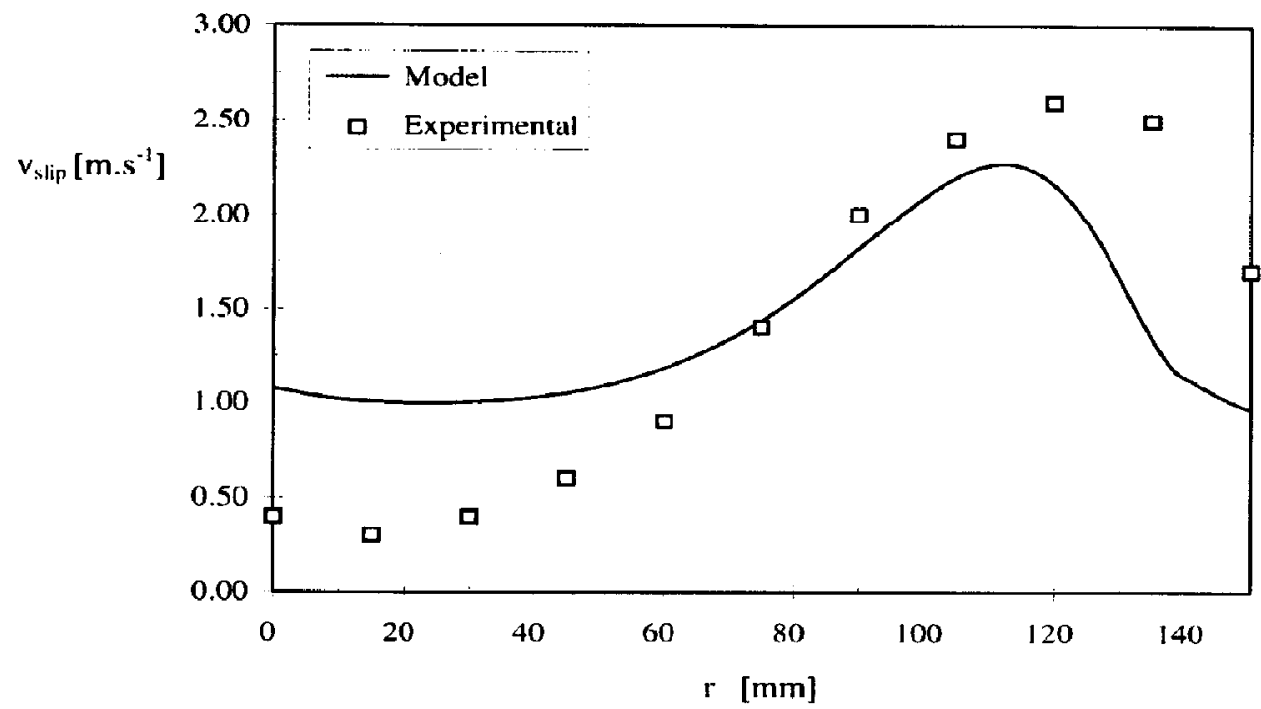

Fig. 8. Comparison of theoretically calculated and experimentally determined (van Breugel et al., 1969a) slip velocity profiles; $D=0.30 \mathrm{~m}, U=6.3 \mathrm{~m} \mathrm{~s}^{-1}, G_{\mathrm{s}}=390 \mathrm{~kg} \mathrm{~m}^{-2} \mathrm{~s}^{-1}, d_{p}=40 \mu \mathrm{m}, \rho_{\mathrm{s}}=2300 \mathrm{~kg} \mathrm{~m}^{-3}$.

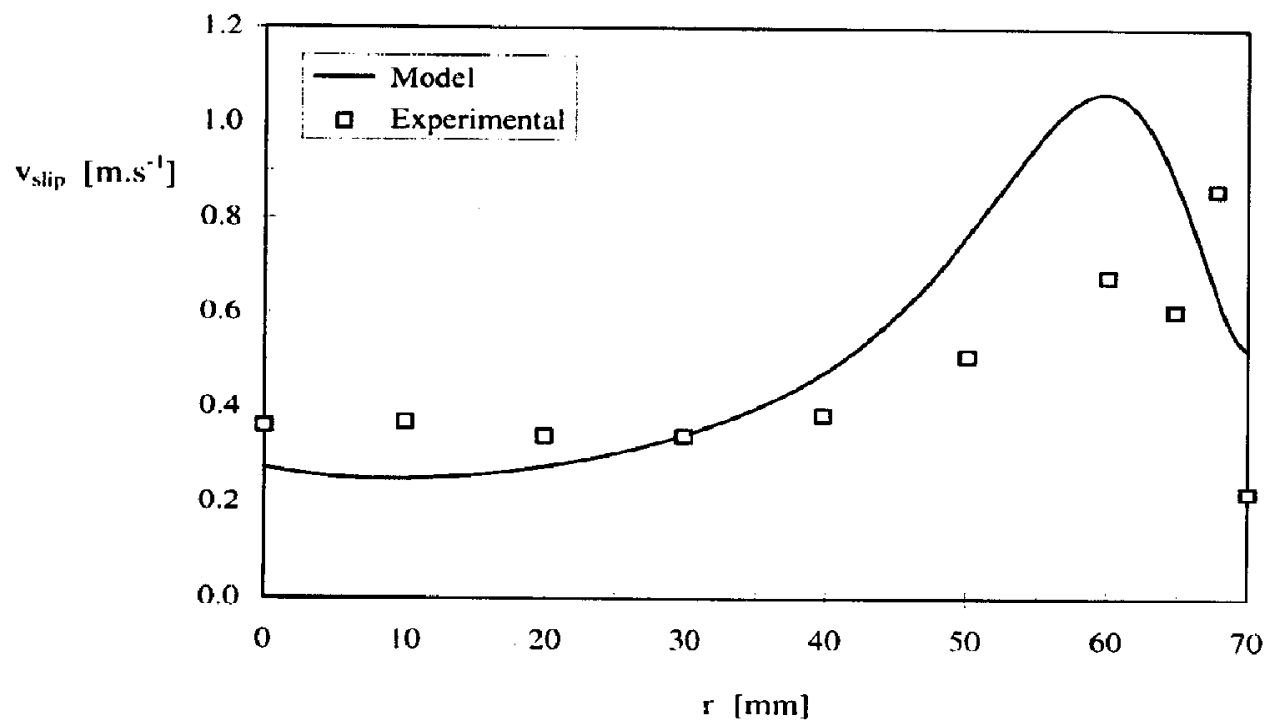

Fig. 9. Comparison of theoretically calculated and experimentally determined (Yang et al., 1992) slip velocity profiles; $D=0.14 \mathrm{~m}, U=4.33 \mathrm{~m} \mathrm{~s}^{-1}, G_{\mathrm{s}}=22 \mathrm{~kg} \mathrm{~m}^{-2} \mathrm{~s}^{-1}, d_{p}=54 \mu \mathrm{m}, \rho_{\mathrm{s}}=1545 \mathrm{~kg} \mathrm{~m}^{-3}$.

transfer coefficient for the existence of clusters of particles.

Near the tube wall the hydrodynamic model predicts the occurrence of a maximum in the slip velocity which is in agreement with experimental observations. This maximum could be explained from detailed consideration of hydrodynamic equations.

Future work will be concerned with the implementation of a collision theory for the particulate phase to predict the radial distribution of solids, eliminating the necessity to specify this distribution using empirical correlations or experimental data.
Acknowledgement-This investigation was financially supported by "Koninklijke Shell Laboratorium Amsterdam" (Shell Research).

\section{NOTATION}

$C_{d, s} \quad$ drag coefficient for an isolated particle

$d_{p} \quad$ diameter, $\mathrm{m}$

$f$ force, $\mathrm{N} \mathrm{m}^{-3}$

$f(\epsilon) \quad$ function, defined in eq. (10)

$G$ solid mass flux, $\mathrm{kg} \mathrm{m}^{-2} \mathrm{~s}^{-1}$

$\mathrm{g}$ acceleration due to gravity, $\mathrm{m} \mathrm{s}^{-2}$

$K$ constant in relation for eddy viscosity 
$L$ tube length, $\mathrm{m}$

$P \quad$ pressure, $\mathrm{kg} \mathrm{m}^{-1} \mathrm{~s}^{-2}$

$R$ tube radius, $\mathrm{m}$

$r$ radial coordinate, $m$

$U$ gas phase velocity in axial direction (superficial), $\mathrm{m} \mathrm{s}^{-1}$

$u$ gas phase velocity in axial direction (interstitial), $\mathrm{m} \mathrm{s}^{-1}$

$v$ solid phase velocity in axial direction (interstitial), $\mathrm{m} \mathrm{s}^{-1}$

$z \quad$ axial coordinate, $m$

\section{Greek letters}

$\beta$ interphase momentum transfer coefficient, $\mathrm{kg} \mathrm{m}^{-3} \mathrm{~s}^{-1}$

$\epsilon$ volume fraction

$\rho$ density, $\mathrm{kg} \mathrm{m}^{-3}$

$\theta$ dimensionless radial coordinate, $r / \boldsymbol{R}$

$\mu \quad$ shear viscosity, $\mathrm{kg} \mathrm{m}^{-1} \mathrm{~s}^{-1}$

\section{Subscripts}

cl cluster

f fluidum

mf minimum fluidisation

p particle

s solid phase

slip velocity difference between both phases

term terminal

w wall

\section{Superscripts}

- averaged over cross-sectional area

c calculated

\section{Dimensionless groups}

$\operatorname{Re}$ Reynolds number $\quad \operatorname{Re}=\frac{\rho_{\mathrm{f}} U D}{\mu_{\mathrm{f}}}$

$R e_{\mathrm{p}}$ particle Reynolds number

$$
\operatorname{Re}_{\mathrm{p}}=\frac{\rho_{\mathrm{f}} \epsilon|\bar{u}-\bar{v}| d_{p}}{\mu_{\mathrm{f}}}
$$

\section{REFERENCES}

Anderson, T, and Jackson, R., 1967, A fluid mechanical description of fluidized beds. Ind. Engng Chem. Fund. 6, $527-539$.

Arastoopour, H. and Gidaspow, D., 1979, Vertical pneumatic conveying using four hydrodynamic models. Ind. Engng Chem. Fund. 18, 123-130.

Bader, R., Findlay, J. and Knowlton, T. M., 1988, Gas/ solids flow patterns in a $30.5 \mathrm{~cm}$. diameter circulating fluidized bed. In Circulating Fluidized Bed Technology II
(Edited by P. Basu and J. F. Large), pp. 123-137. Pergamon Press, Oxford.

van Breugel, J. W., Stein, J. J. M. and Buurman, C., 1969a, Vertical gas solids flow. Internal report Shell Laboratory Amsterdam, The Netherlands.

van Breugel, J. W., Stein, J. J. and de Vries, R. J., 1969b, Isokinetic sampling in a dense gas solids stream, Patent 3C. Proc. Inst. Mech. Engng 184, 18-21.

Deissler, R. G., 1955, NACA report 1210.

Ding, J, and Gidaspow, D., 1990. A bubbling fluidization model using kinetic theory of granular flow. A.I.Ch.E. J. 36, 523-538.

Dry, R. J., 1986, Radial concentration profiles in a fast fluidised bed. Power Technol. 49, 37-44.

Gidaspow, D., Tsuo, Y. P. and Luo, K. M., 1989, Computed and experimental cluster formation and velocity profiles in circulating fluidized beds. In Fluidization VI (Edited by J. R. Grace, L. W. Shemilt and M. A. Bergougnou), pp. $81-88$

Grace, J. R., 1970, The viscosity of nuidised beds. Can J. Chem. Engng 48, 30-33.

Grace, J. R. and Tuot, J., 1979, A theory for cluster formation in vertically conveyed suspensions of intermediate density. Trans. Ind. Chem. Engng 57, 49-54.

Kwauk, M., Ningde, W., Youchu, L. and Bingyu, C., 1986, Fast fluidization at ICM. In Circoloting Fhidized Bed Technology I (Edited by P. Basu), pp. 33-61.

Matsen, J. M., 1982, Mechanisms of choking and entrainment. Powder Technol. 32, 21-33.

Miller, A. and Gidaspow, D., 1992, Dense vertical gas solid flow in a pipe. A.I.Ch.E.J. 38 (11), 1801-1815.

Monceaux, L., Azzi, M., Molodtsof, Y. and Large, J. F., 1986, Overall and local characterisation of flow regimes in a circulating fluidized bed. In Circulating Fluidized Bed Technology I (Edited by P. Basu), pp. 185-191.

Rhodes, M., Zhou, S. and Benkreira, H., 1992, Flow of dilute gas particle suspensions. A.I.Ch.E. J. 38 (12), 1913-1915.

Schiller, L. and Naumann, A., 1935, Über die grundlegenden Berechnungen bei der Schwerkraftaufbereitung. $\mathcal{Z}$. Ver. Disch. Ing. 77, 318-326.

Siemes, W. and Hellmer, L., 1962, Die Messung der Wirbelschichtsviskosität mit der pneumatischen Rinne. Chem. Engng Sci. 17, 555571.

Tsuji, Y., Morikawa, Y. and Shiomi, H., 1984, LDV measurements of an air-solid flow in a vertical pipe. J. Fluid Mech. 139, $417-434$.

Wen, Y. C. and Yu, Y. H., 1966, Mechanics of fluidization. Chem. Engng Prog. Symp. Ser. 62 (62), 100-111.

Yang, Y. L., Yin, Y., Yu, Z. Q. and Wang, Z. W., 1992, Investigation on slip velocity distributions in the riser of dilute circulating fluidized beds. Powder Technol. 73, 6773.

Yerushalmi, J. and Avidan, A. A., 1985, High velocity fluidization. In Fluidization, 2nd Edn (Edited by J. F. Davidson and D. Harrison), pp. 225-291. Academic Press, New York.

Yerushalmi, J., Cankurt, N. T., Geldart, D. and Liss, B., 1978 , Flow regimes in vertical gas-solid contact systems. A.I.ChE. Symp. Ser. 74 (1974), 1-13.

Zhang, W., Tung, $Y$. and Johnsson, $F_{+}$, 1991, Radial voidage profiles in fast fluidized beds of different diameters. Chem. Engng Sci. 46 (12), 2045-3052. 